\title{
Sulphur and Molybdenum Incorporation at the Calcite-Water Interface: Insights from Ab Initio Molecular Dynamics
}

\author{
Scott D. Midgley, ${ }^{\text {a Devis Di Tommaso, }},{ }^{\mathrm{b}}$ Dominik Fleitmann, ${ }^{\mathrm{c}, \mathrm{d}}$ Ricardo Grau-Crespo ${ }^{\mathrm{a}, *}$ \\ a Department of Chemistry, School of Chemistry, Food and Pharmacy, University of Reading, United Kingdom, RG6 6DX. Email: r.grau- \\ crespo@reading.ac.uk \\ ${ }^{b}$ Thomas Young Centre, Materials Research Institute, School of Biological and Chemical Sciences, Queen Mary University of London, Mile End Road, \\ London, United Kingdom, E1 4NS \\ ${ }^{c}$ Department of Archaeology, School of Archaeology, Geography and Environmental Science, University of Reading, United Kingdom, RG6 6AD \\ ${ }^{d}$ Department of Environmental Sciences, University of Basel, Switzerland.
}

\begin{abstract}
Sulphur and molybdenum trace impurities in speleothems (stalagmites and stalactites) can provide long and continuous records of volcanic activity, which are important for past climatic and environmental reconstructions. However, the chemistry governing the incorporation of the trace-element bearing species into the calcium carbonate phases forming speleothems is not well understood. Our previous work has shown that substitution as tetrahedral oxyanions $\left[\mathrm{XO}_{4}\right]^{2-}\left(\mathrm{X}=\mathrm{S}\right.$, Mo) replacing $\left[\mathrm{CO}_{3}\right]^{2-}$ in $\mathrm{CaCO}_{3}$ bulk phases (except perhaps for vaterite) is thermodynamically unfavourable with respect to the formation of competing phases, due to the larger size and different shape of the $\left[\mathrm{XO}_{4}\right]^{2-}$ tetrahedral anions in comparison with the flat $\left[\mathrm{CO}_{3}\right]^{2-}$ anions, which implied that most of the incorporation would happen at the surface rather than the bulk of the mineral. Here we present an ab initio molecular dynamics study exploring the incorporation of these impurities at the mineralwater interface. We show that the oxyanions substitution at the aqueous calcite (10.4) surface is clearly favoured over bulk incorporation, due to the lower structural strain on the calcium carbonate solid. Incorporation at surface step sites is even more favourable for both oxyanions, thanks to the additional interface space afforded by the surface line defect to accommodate the tetrahedral anion. Differences between sulphate and molybdate substitution can be mostly explained by the size of the anions. The molybdate oxyanion is more difficult to incorporate in the calcite bulk than the smaller sulphate oxyanion. However, when molybdate is substituted at the surface, the elastic cost is avoided because the oxyanion protrudes out of the surface and gains stability via the interaction with water at the interface, which in balance results in more favourable surface substitution for molybdate than for sulphate. The detailed molecular-level insights provided by our calculations will be useful to understand the chemical basis of S- and Mo-based speleothem records.
\end{abstract}

Keywords: calcite, sulphate, molybdate, stalagmites, volcanic activity records, ab initio molecular dynamics 


\section{Introduction}

Paleoclimate reconstructions are essential for understanding modern-day climate change, but are hindered by a lack of near-instrumental environmental records. Long and continuous climate reconstructions therefore rely upon geological archives (e.g., marine and lake sediments, trees, speleothems) of prevailing environmental conditions in the past. Input parameters for climate models simulating past climate variability require information obtained from various geological phenomena, including volcanic eruptions, which can trigger global cooling by disrupting natural transfer of solar radiation when volcanic material enters the atmosphere. ${ }^{1-2}$ Identifying the magnitude of volcanic activity that occurred before modern scientific and historical records, involves examining geological archives for chemical indicators (proxies). Ice cores, ${ }^{3-5}$ tree rings, ${ }^{6}$ and marine sediments ${ }^{7}$ are examples of widely exploited geological archives, which have the potential to capture and preserve geochemical indicators of volcanic activity. These archives hold geochemical proxies in naturally undisturbed states for millennia, allowing for environmental indicators to be matched to specific periods of Earth's history. In recent years, speleothems have seen increased research interest for use as a new paleo-volcanic archive..$^{8-11}$ These calcium carbonate mineral structures are highly stable and have advantages over ice cores, due to their usual proximity in relation to many volcanic sites. Speleothems form in discrete layers primarily from cave drip-water. Chemical impurities associated with geological and atmospheric conditions following a volcanic eruption can be detected in radiometrically dated subsections of speleothem mineral. ${ }^{12-13}$ Prevalence of certain impurities can be indicative of an environmental anomaly, such as alteration of atmospheric chemistry from a volcanic eruption. For example, Badertscher and co-workers have successfully matched elevated levels of sulphur, molybdenum and bromine to the well-known Minoan volcanic eruption that occurred on Santorini, around 1600 BCE. ${ }^{8}$ In this study, trace amounts of these elements were measured in speleothem samples by X-ray fluorescence spectroscopy. A quantitative link between measured concentrations of impurity and properties of the volcanic eruption, e.g. eruption magnitude or proximity to the speleothem, has not been established so far. The exact chemical nature of the trace-element bearing species is still not well understood either, though the chemical form and mechanism of incorporation are likely to be important considerations to improve the interpretation of trace element records obtained from speleothems.

The calcium carbonate in speleothems is mainly in calcite form, with aragonite also present in some cases. ${ }^{14-16}$ Other calcium carbonate phases can also be found in speleothems but are very rare. Recently, we used computer simulations to study the lattice incorporation of both sulphur and molybdenum in the form of tetrahedral molybdate anions, in all the naturally occurring bulk phases of calcium carbonate. ${ }^{17} \mathrm{We}$ found that both sulphate and molybdate anions are unstable in the bulk of calcite, aragonite or vaterite, with respect to the formation of naturally occurring competing phases. In the case of sulphate ions, only the least dense vaterite polymorph provides a somewhat stable host for lattice substitution at low concentrations. A preliminary calculation of substitution at the calcite (10.4) surface (ignoring dynamic aspects and assuming a simple interface with vacuum) showed that substitution at the surface, where there is more space to accommodate the tetrahedron anion, is energetically more favourable than in the bulk. ${ }^{17}$ If indeed trace elements are mainly 
incorporated at the surface or grain boundaries, then the crystallinity of the calcium carbonate in the stalagmite will directly impact detected trace-element concentrations. This effect is important to interpret the speleothem record and requires a more careful and detailed investigation, considering some of the complexity of mineral surfaces and interfaces, which is the purpose of the present work.

The (10.4) surface of calcite investigated here is very stable and typically the most prominent in natural calcite particle morphology, ${ }^{18-20}$ making it a good representative surface for the present study. Calcite growth occurs through the formation and motion of monolayer steps at the surfaces. ${ }^{21-22}$ This growth is well known to be inhibited by the incorporation of cation impurities (most notably $\mathrm{Mg}^{2+}$ ) at the steps. ${ }^{23-26}$ Meyer and subsequent citing articles have indicated that the sulphate anion is also a calcite growth inhibitor. ${ }^{25}$ It is therefore clear that these steps can provide a more favourable environment for the incorporation of impurities than the bulk or the perfect surfaces. ${ }^{27-28}$ We report here density functional theory (DFT) molecular dynamics simulations of the incorporation of sulphate and molybdate oxyanions both at terrace and step sites of the calcite (10.4) surface.

\section{Methodology}

The bulk phase of calcite, a trigonal crystal with space group of R-3C (167) ${ }^{29}$ was modelled using a $3 \times 3 \times 1$ supercell of the hexagonal unit cell, to minimise interactions between trace elements and their periodic images. Periodic slab models were used to represent the calcite/water interface. The slab for the (10.4) perfect surface consisted of four $\mathrm{CaCO}_{3}$ layers in the crystallographic direction perpendicular to the surface. The gap between slabs was filled with water molecules at a density of $1 \mathrm{~g} \mathrm{~cm}^{-3}$. We also considered 'imperfect' or stepped surfaces, to represent formation of pits on the calcite (10.4) growth termination. To represent this in the (10.4) surface models, we created a vicinal (31.8) surface, giving an acute step in the standard (10.4) terrace. Other types of step such as those with an obtuse angle are known, but are less likely to show long term stability with respect to dissolution. ${ }^{30}$

Three compositions were investigated in each slab model: pure calcium carbonate, calcium carbonate with $\left(\mathrm{SO}_{4}\right)^{2-} /\left(\mathrm{CO}_{3}\right)^{2-}$ substitution, and calcium carbonate with $\left(\mathrm{MoO}_{4}\right)^{2-} /\left(\mathrm{CO}_{3}\right)^{2-}$ substitution. In each substituted case, a single carbonate ion was replaced with the relevant trace-element containing oxyanion. At the perfect surface, the substitution was performed at the top layer, at the solid/water interface. At the stepped surface, we considered substitution only at the apex of the step as this has most space to accommodate the ion impurity, maximising the thermodynamic benefits of this surface topography. The vicinal surface cleavage left two symmetrically inequivalent sites at the apex of the step, which we called $\mathrm{C} 1$ and $\mathrm{C} 2$ arbitrarily. Initial investigation revealed negligible energetic differences between these two anion sites, therefore we proceeded to consider just the $\mathrm{C} 1$ site. As a reference phase, each anion (carbonate, sulphate and molybdate) was placed into a ca. $15 \AA$ box containing water molecules at a density of $1 \mathrm{~g} \mathrm{~cm}^{-3}$. Each anion in a box of water introduces a double negative charge to simulation cell; this was corrected using a homogeneous charge background.

The ab initio molecular dynamics (AIMD) simulations were performed at constant volume and temperature using the canonical NVT ensemble implemented in the CP2K software package. ${ }^{31}$ To find the 
equilibrium volume and cell parameters at $300 \mathrm{~K}$, while avoiding a computationally expensive constant-pressure (NPT) simulation at ab initio level, we followed a two-step procedure. First, we optimised the bulk structure statically (i.e. at $0 \mathrm{~K}$ and ignoring zero-point effects) using $\mathrm{CP} 2 \mathrm{~K}$. To calculate the temperature-correction to the $0 \mathrm{~K}$ cell parameters, we then performed NPT classical molecular dynamics (MD) simulations at $300 \mathrm{~K}$, using a calcite $3 \times 3 \times 1$ bulk supercell. The classical simulations were carried out using the GULP code ${ }^{32}$ with the carbonates forcefield library by Fisler et al.. ${ }^{33}$ Cell vectors simulated in GULP at $0 \mathrm{~K}$ and $300 \mathrm{~K}$ were used to calculate the average linear thermal expansion from $0 \mathrm{~K}$ to $300 \mathrm{~K}$, which was then applied as a correction to the cell vectors optimised at $0 \mathrm{~K}$ using the $\mathrm{CP} 2 \mathrm{~K}$ code. Table 1 shows that the predicted cell parameters are in reasonably good agreement with crystallographic measurements. The negative thermal expansion in the $a$-axis is consistent with previous reports. ${ }^{34}$

Table 1: Calcite cell vector lengths obtained from $\mathrm{CP} 2 \mathrm{~K}$ at $0 \mathrm{~K}$, and after finite temperature correction, in comparison with experimental values.

\begin{tabular}{rrrr}
\hline & $\boldsymbol{T}=\mathbf{0 ~ K}$ & $\boldsymbol{T}=\mathbf{3 0 0 K}$ & Exp. $(\mathbf{2 9 7} \mathbf{~ K})^{29}$ \\
\hline $\boldsymbol{a}(\AA)$ & 5.22 & 5.07 & 4.99 \\
$\boldsymbol{c}(\AA)$ & 17.59 & 17.74 & 17.06 \\
\hline
\end{tabular}

In the AIMD simulations, electronic minimisation was carried out using the Quickstep implementation of DFT, ${ }^{35}$ where the orbital transformation method was employed. ${ }^{36}$ All DFT calculations used the generalised gradient approximation (GGA) in the form of the revised Perdew-Burke-Ernzerhof (revPBE) exchangecorrelation functional. ${ }^{37}$ The revPBE functional has been shown to be particularly effective for describing the liquid structure of water, especially compared to the standard PBE functional. ${ }^{38}$ For representation of dispersion corrections, which is particularly important when simulating liquids, Grimme's D3 corrections were applied. ${ }^{39}$ In all calculations the short ranged, double-zeta, molecule-optimised basis sets were used with polarisation on heavy atoms (DZVP-MOLOPT-SR-GTH). ${ }^{40}$ The Goedecker-Teter-Hutter (GTH) pseudopotentials were used to represent atomic cores. ${ }^{41}$ The Nose-Hoover thermostat was used for the NVT simulations, with a time constant of $13 \mathrm{fs} .{ }^{42}$ A time step of $1 \mathrm{fs}$ was used in all simulations, with the initial temperature set to $300 \mathrm{~K}$. Each interface system was subject to ca. 120,000 steps, giving a total simulation time of $120 \mathrm{ps}$. For the ab initio MD simulations of $E\left[\mathrm{Ca}_{n}\left(\mathrm{CO}_{3}\right)_{(n-1)} X \mathrm{O}_{4}\right]_{(\text {slab,aq })}$ each time step required, on average, around 6-8 seconds on 576 cores of the UK National Supercomputing Service ARCHER. The ab initio MD simulation of interfaces models reported herein required in the region of 134,000 CPU hours (wall-clock time $\times$ number of processors).

We characterize the thermodynamics of surface incorporation using three interdependent parameters (any two of them determine the value of the third). The first one is the surface/liquid exchange energy, which is the energy required to exchange one carbonate and one impurity anion between the surface and the aqueous phase: 


$$
\begin{aligned}
\Delta E_{\text {exch }}^{\text {surf }}=E & {\left[\mathrm{Ca}_{n}\left(\mathrm{CO}_{3}\right)_{n-1} X \mathrm{O}_{4}\right]_{(\text {slab,aq })}+E\left[\left(\mathrm{CO}_{3}\right)^{2-}\right]_{(\mathrm{isol}, \mathrm{aq})} } \\
& -\left(E\left[\mathrm{Ca}_{n}\left(\mathrm{CO}_{3}\right)_{n}\right]_{(\text {slab,aq })}+E\left[\left(\mathrm{XO}_{4}\right)^{2-}\right]_{(\text {isol,aq })}\right)
\end{aligned}
$$

where $E\left[\mathrm{Ca}_{n}\left(\mathrm{CO}_{3}\right)_{n-1} X \mathrm{O}_{4}\right]_{(\mathrm{slab}, \mathrm{aq})}$ is the energy of the calcite/water slab model containing one $\left(X \mathrm{O}_{4}{ }^{2-}\right)$ anion in a carbonate position, $E\left[\mathrm{Ca}_{n}\left(\mathrm{CO}_{3}\right)_{n}\right]_{(\mathrm{slab}, \mathrm{aq})}$ is the energy of the pure calcite/water slab, and

$E\left[\left(\mathrm{CO}_{3}\right)^{2-}\right]_{(\mathrm{isol}, \mathrm{aq})}$ and $\left.E\left[\left(\mathrm{XO}_{4}\right)^{2-}\right]_{(\mathrm{isol}, \mathrm{aq})}\right)$ are the energies of the isolated anions in water. In our calculations, there are $n=48$ formula units in the non-defective surface slab supercell and $n=52$ formula units in the stepped surface supercell.

The second calculated parameter is the bulk/liquid exchange energy - the energy required to exchange one carbonate/trace ion from the calcite bulk with aqueous phase:

$$
\begin{aligned}
\Delta E_{\mathrm{exch}}^{\mathrm{bulk}}= & E\left[\mathrm{Ca}_{m}\left(\mathrm{CO}_{3}\right)_{m-1} X \mathrm{O}_{4}\right]_{(\mathrm{bulk})}+E\left[\left(\mathrm{CO}_{3}\right)^{2-}\right]_{(\mathrm{isol}, \mathrm{aq})} \\
& -\left(E\left[\mathrm{Ca}_{m}\left(\mathrm{CO}_{3}\right)_{m}\right]_{(\mathrm{bulk})}+E\left[\left(\mathrm{XO}_{4}\right)^{2-}\right]_{(\mathrm{isol}, \mathrm{aq})}\right)
\end{aligned}
$$

where $E\left[\mathrm{Ca}_{m}\left(\mathrm{CO}_{3}\right)_{m-1} X \mathrm{O}_{4}\right]$ is the energy of the calcite bulk $3 \times 3 \times 1$ supercell model containing one $\left(X \mathrm{O}_{4}{ }^{2-}\right)$ anion in a carbonate position, $E\left[\mathrm{Ca}_{m}\left(\mathrm{CO}_{3}\right)_{m}\right]$ is the energy of the pure calcite unsubstituted supercell. Finally, the segregation energy, which is the energy required to exchange one carbonate/trace ion between the bulk and the (hydrated) surface of calcite, is given by:

$$
\begin{gathered}
\Delta E_{\text {seg }}=E\left[\mathrm{Ca}_{n}\left(\mathrm{CO}_{3}\right)_{n-1} X \mathrm{O}_{4}\right]_{(\text {slab }, \mathrm{aq})}+E\left[\mathrm{Ca}_{m}\left(\mathrm{CO}_{3}\right)_{m}\right]_{(\text {bulk })} \\
-\left(E\left[\mathrm{Ca}_{n}\left(\mathrm{CO}_{3}\right)_{n}\right]_{(\text {slab,aq })}+E\left[\mathrm{Ca}_{m}\left(\mathrm{CO}_{3}\right)_{m-1} X \mathrm{O}_{4}\right]_{(\text {bulk })}\right) \\
=\Delta E_{\text {exch }}^{\text {surf }}-\Delta E_{\text {exch }}^{\text {bulk }}
\end{gathered}
$$

The absolute energies used in equations 1-3 to define $\Delta E_{\mathrm{exch}}^{\text {surf }}, \Delta E_{\mathrm{exch}}^{\mathrm{bulk}}$, and $\Delta E_{\text {seg }}$ were obtained from the average energies of the MD simulations after equilibration. Equilibrated sections of the MD simulation were found to be around the second half of the MD simulations, ca. 90-100 ps of MD run time. In most cases, the first 20 ps of the MD simulation involved equilibration, meaning that the production period consists of ca. 90-100 ps of MD run time in all cases.

\section{Results and discussion}

Table 2 summarises the exchange and segregation energies obtained for molybdate and sulphate substitutions in calcite. The bulk/liquid exchange energies in calcite $(1.08 \mathrm{eV}$ and $1.62 \mathrm{eV}$ for sulphate and molybdate, respectively) show the same trend to the bulk/vacuum exchange energies reported in our previous work (1.15 eV and $1.45 \mathrm{eV}$, respectively), ${ }^{17}$ i.e. molybdate substitution incurred a greater energy penalty than sulphate substitution, due to the larger size of the former anion. Though the trend in the substitution energies was the same, the difference between sulphate and molybdate substitution was found here to be different (larger) than what was reported in Ref 17 . This is partly because we now use a different thermodynamic reference phase, 
i.e. oxyanions in water instead of oxyanions in vacuum, and partly because of the dynamic nature of the simulations here, in contrast with the static calculations in Ref 17 . We will see below that, indeed, the incorporation of oxyanion impurities in calcite contains distinctive dynamic features that cannot be captured by static calculations, even for bulk substitution.

Table 2. Exchange and segregation energies of molybdate and sulphate species in calcite.

\begin{tabular}{cccccc}
\hline \multirow{2}{*}{ Species } & $\begin{array}{c}\Delta E_{\text {exch }}^{\text {bulk }} \\
(\mathbf{e V})\end{array}$ & \multicolumn{2}{c}{$\Delta E_{\text {exch }}^{\text {surf }}$} & \multicolumn{2}{c}{$\Delta E_{\text {seg }}$} \\
& bulk & $(\mathbf{e V})$ & & \multicolumn{2}{c}{$(\mathbf{e V})$} \\
\cline { 2 - 7 } & & terrace & step & terrace & step \\
\hline sulphate & 1.08 & 0.79 & 0.27 & -0.29 & -0.81 \\
molybdate & 1.62 & 0.48 & -0.07 & -1.14 & -1.69 \\
\hline
\end{tabular}

Exchange energies at the (10.4) terrace are reduced when compared to equivalent bulk exchange energies, indicating overall greater thermodynamic stability of the impurities at the surface. Unlike the bulk exchange process, molybdate incorporation at the terrace is more favourable than sulphate, which is surprising when considering that the former is a much larger species. These observations will be rationalized below, based on the discussion of the geometries of the substituted structures. Exchange energies at the step are even more favourable than at the terrace, indicating further thermodynamic favourability than bulk and terrace substitution. Like at the terrace, molybdate substitution is favoured over sulphate substitution at the step, with a comparable energy difference of ca. $0.3 \mathrm{eV}$.

Overall, ion exchange in calcite with sulphate and molybdate follows the order: stepped surface > terrace > bulk. The tetrahedral oxyanion impurity does not fit the planar carbonate anion site in calcite. As a result, significant lattice strain is caused in the bulk, which is reduced at surface regions due to the increased interfacial space around the substitution site. The step affords even greater interfacial space than the terrace, which is why the trend in exchange energies at the bulk, terrace and step follow the expected order. The crystallinity of the speleothem and factors affecting the stability of imperfect calcite surface topographies may therefore have a significant impact on detected concentrations of these trace elements in speleothems. Therefore, this should be an important consideration for the interpretation of impurity concentration data collected from speleothems.

To get further insights and rationalise the results from the thermodynamics analysis, we now discuss the MD trajectories and average geometries in equilibrium. Figure 1 shows snapshots from the MD simulations, taken after equilibration and at energies close to the equilibrium average. Fig. 2 a and b illustrate why the bulk of calcite is not well suited to accommodate the large tetrahedral oxyanion species, as the apical oxygen atom is unable to fit within the planar carbonate anion site. Interestingly, the tetrahedral oxyanions is found to rotate 
significantly with respect to the plane of the carbonate anions. For example, in the snapshot of the simulation of sulphate in bulk calcite (Fig. 2a), two of the four oxygen atoms occupy the same oxygen positions as in the vacant carbonate ion, whereas the other two oxygen atoms are above and below the substituting plane. This contrasts with the equilibrium geometry found in our static DFT work, ${ }^{17}$ where three of the sulphate oxygen atoms occupied the three vacant carbonate oxygen sites, and the fourth (apical) sulphate oxygen pointing out of the plane above the incorporation site. The observation of oxyanion rotation suggests that static calculations are not fully able to capture the behaviour of tetrahedral oxyanion dopants in calcite, even for bulk simulations. This is important because static DFT (or forcefield-based) calculations are still the most widely used method for the simulation of impurities in carbonate minerals.

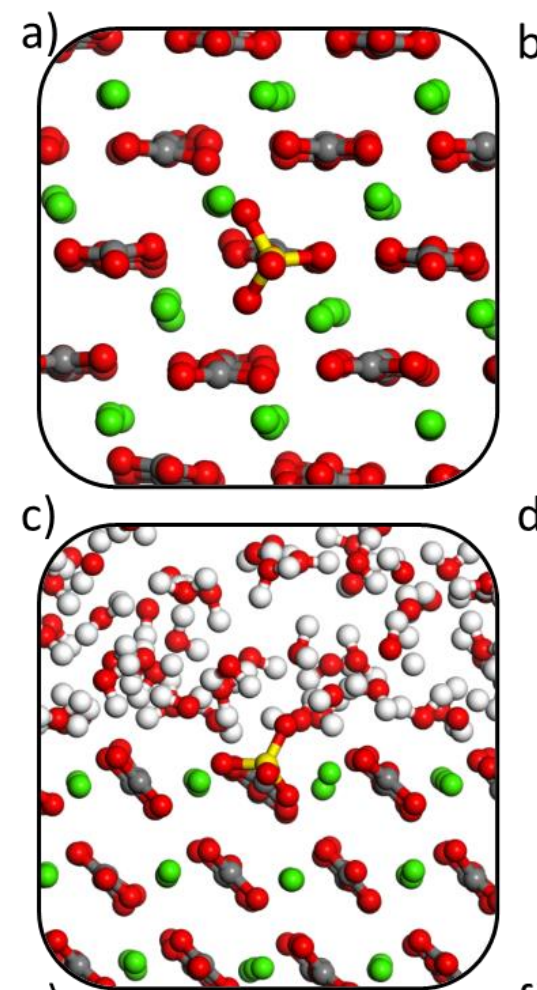

e)

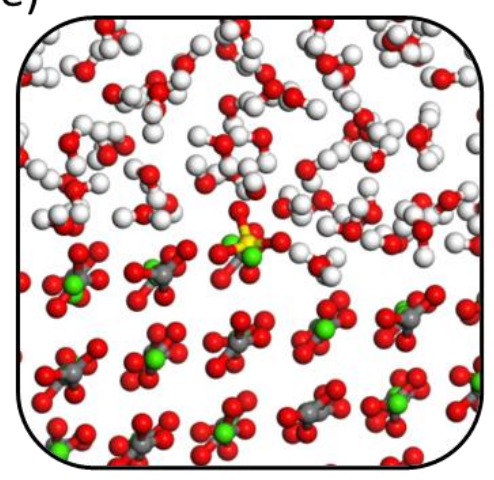

b)

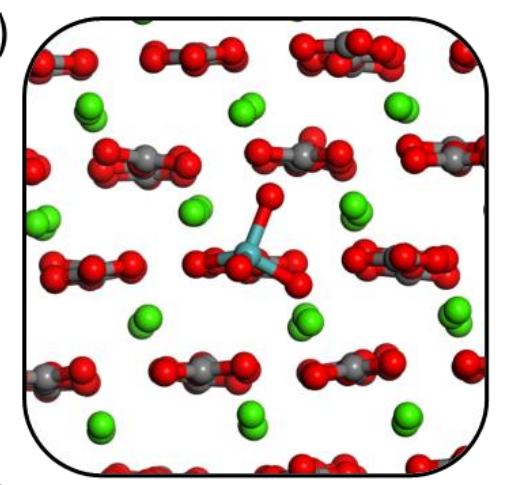

d)

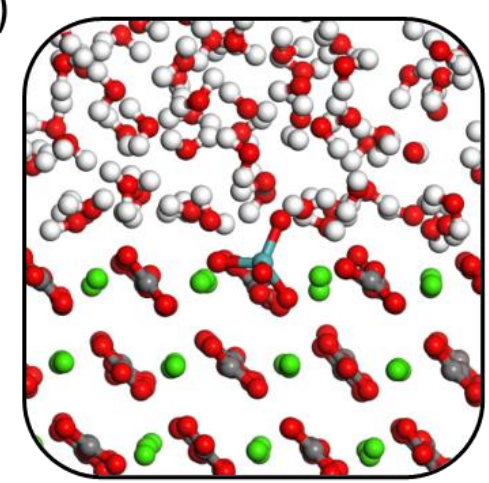

f)

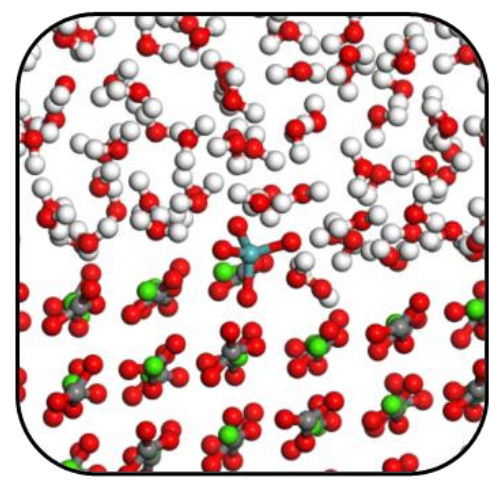

Figure 1. MD trajectory snapshots for calcite substituted with a) sulphate at the bulk, b) molybdate at the bulk, c) sulphate at the terrace, d) molybdate at the terrace, e) sulphate at the step, f) molybdate at the step. 
In contrast with the bulk substitution, when the oxyanion impurities are substituted at the surface, the apical oxygen atom can stick out, which not only decreases the elastic cost of the substitution, but also leads to extra stabilization by a favourable interaction with water at the interface. To describe this effect in a more quantitative way, we turn to the calculated radial distribution functions (RDFs) from the MD simulations. The RDF $g(r)$ is defined in such a way that the number of atoms of the given type at a distance between $r$ and $r+\mathrm{d} r$ from the central atom is proportional to $\mathrm{g}(r) \mathrm{d} r$, and the function is normalised so that $\mathrm{g}(r)=1$ when $r \rightarrow \infty$.

We start with the analysis of RDF's for $X-\mathrm{O}_{\mathrm{w}}$ (i.e. from $\mathrm{C}, \mathrm{S}$ or Mo atom in the oxyanion to the oxygen atoms $\mathrm{O}_{\mathrm{w}}$ in water molecules), for the simulation of isolated oxyanions in water are reported in Figure 2a. The three anions have the same charge but different ion sizes (carbonate $<$ sulphate $<$ molybdate), as illustrated by the peak positions between 3 and $4 \AA$ in the $X-\mathrm{O}_{\mathrm{w}} \mathrm{RDFs}$. This means that the strength of the interaction with water is expected to follow the opposite trend (carbonate > sulphate > molybdate), in agreement with experimental hydration enthalpies, e.g. the hydration of a carbonate anion is $\sim 2.6 \mathrm{eV}$ more exothermic than the hydration of the larger sulphate ion. ${ }^{43}$ Compared with the case of sulphate, the first hydration sphere around molybdate is shifted right and broadened. There is also no-clearly defined second hydration sphere, proving that polarization on the surrounding water is much weaker for molybdate. This observation is consistent with our calculation of the mean residence time (MRT) of water molecules surrounding the isolated sulphate and molybdate ions. These are obtained as $\mathrm{MRT}=t_{\mathrm{sim}} \times \mathrm{CN}_{\mathrm{ave}} / N_{\mathrm{ex}}$, where $t_{\mathrm{sim}}$ is the ab initio MD simulation time, $\mathrm{CN}_{\text {ave }}$ is the mean coordination number for sulphate and molybdate, and $N_{\mathrm{ex}}$ is the number of exchange events involving the first hydration sphere during the simulation time (only those with a duration longer than $0.5 \mathrm{ps}$ are counted). MRTs for sulphate and molybdate were calculated as 0.8 and 0.5 ps respectively, which indicate that the hydration environments of both oxyanions are highly labile (comparable MRTs to that of the $\mathrm{Cl}^{-}$ion) ) $^{44}$ but more so for molybdate than for sulphate. 

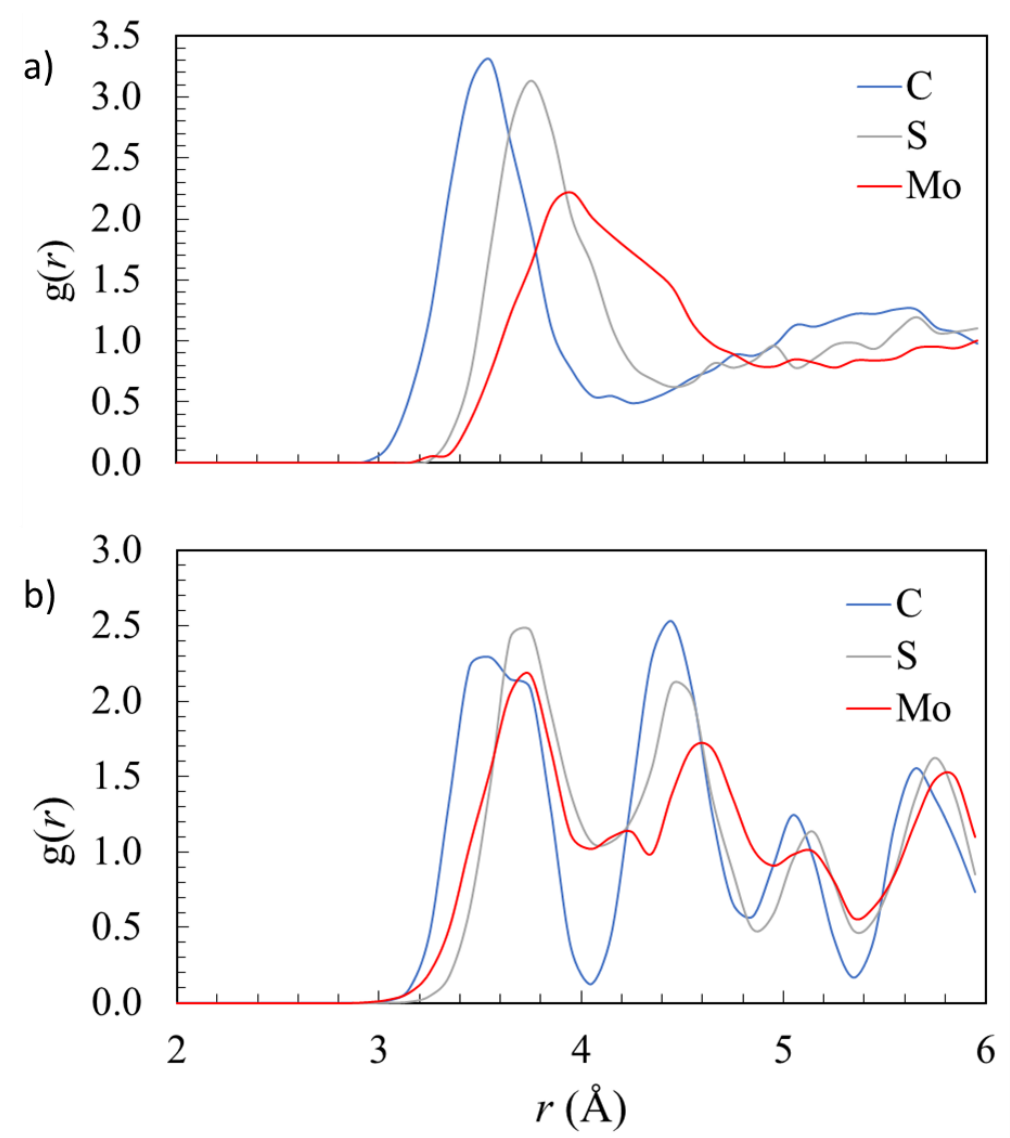

Figure 2. a) Radial distribution function $X$ - $\mathrm{O}_{\mathrm{w}}$, i.e. from the $X=(\mathrm{C}, \mathrm{S}, \mathrm{Mo})$ atom in the $\left(X \mathrm{O}_{n}\right)^{2-}$ oxyanion to the oxygen atoms $\left(\mathrm{O}_{\mathrm{w}}\right)$ in water molecules, for the simulation of isolated oxyanions in water, $\left.b\right)$ Radial distribution function $X-\mathrm{O}_{\mathrm{c}}$, i.e. from the $X=(\mathrm{C}, \mathrm{S}, \mathrm{Mo})$ atom in the $\left(X \mathrm{O}_{n}\right)^{2-}$ oxyanion to the oxygen atom $\left(\mathrm{O}_{\mathrm{c}}\right)$ of the carbonate anions, for the simulation of bulk calcite.

The $X-\mathrm{O}_{\mathrm{c}} \mathrm{RDFs}$, i.e. from the $X=(\mathrm{C}, \mathrm{S}, \mathrm{Mo})$ atom in the $\left(X \mathrm{O}_{n}\right)^{2-}$ oxyanion to the oxygen atom $\left(\mathrm{O}_{\mathrm{c}}\right)$ of the carbonate anions, for the simulation of bulk calcite is shown in Figure $2 \mathrm{~b}$. The first peak at around $3.5 \AA$ corresponds to $X$-O distances in the monolayer above and below the plane of the anion, and this clearly illustrates the structural impact of the impurity on the surrounding ions. Peaks for sulphate and molybdate are shifted right compared with carbonate, which is consistent with crystallographic strain and repulsion with neighbouring carbonate ions, caused by the tetrahedral impurities. The second peak at around $4.5 \AA$ corresponds to the $X$-O distances in the neighbouring carbonate ions in the plane of the subject anion. Here, the sulphur and molybdenum peaks are shifted right, again indicating crystallographic strain and repulsion of the neighbouring carbonate ions by the presence of the impurity. Where $X=$ Mo, oxygen atoms are repelled most significantly, due to the large size of the molybdate anion.

At the surface, the unfavourable steric strain in the lattice caused by tetrahedral oxyanion substitution is reduced, as the apical oxygen atom can protrude from the solid surface rather than causing strain on neighbouring ions in the crystal. This effect is further extended at the step where the substituting anion is afforded maximal interfacial space, meaning very low structural strain the carbonate solid. At the step, three out 
of four oxygen atoms can protrude from the carbonate solid, while still allowing the anion to be chemically bound. This explains why overall, the surface substitutions are more favourable than the equivalent bulk substitutions.

Comparison of RDF's for molybdate and sulphate at the terrace and step may be made from figures $3 \mathrm{a}$ and $3 b$, which explain the lower exchange energies for both ions at the step than the terrace, as reported in Table 2. When substituting the impurity at the terrace, there is around $180^{\circ}$ of arc which is in contact with the water (the other $180^{\circ}$ is in contact with the lattice). When substituting at the step however, the angle of arc in contact with the water is around $270^{\circ}$, which allows for the formation of larger partial hydration spheres, which drive thermodynamic favourability. We discuss molybdenum as an example (sulphate may be assumed to behave in the same way, but with effects less pronounced because of its smaller size). RDFs reported in Figure 3 show the partial hydration environment when molybdate is incorporated at the terrace and the step. From the figure 3, the first hydration sphere around molybdate at $3.8 \AA$ is clearly better defined at the step (4b) than at the terrace (4a). This is because the molybdate ion at the step has more contact with liquid water, causing greater polarization of the aqueous layer and more favourable partial hydration spheres. A combination of decreased lattice strain and increased hydration sphere formation at the step when compared to the terrace explain why there is a thermodynamic preference for substitution at the step.
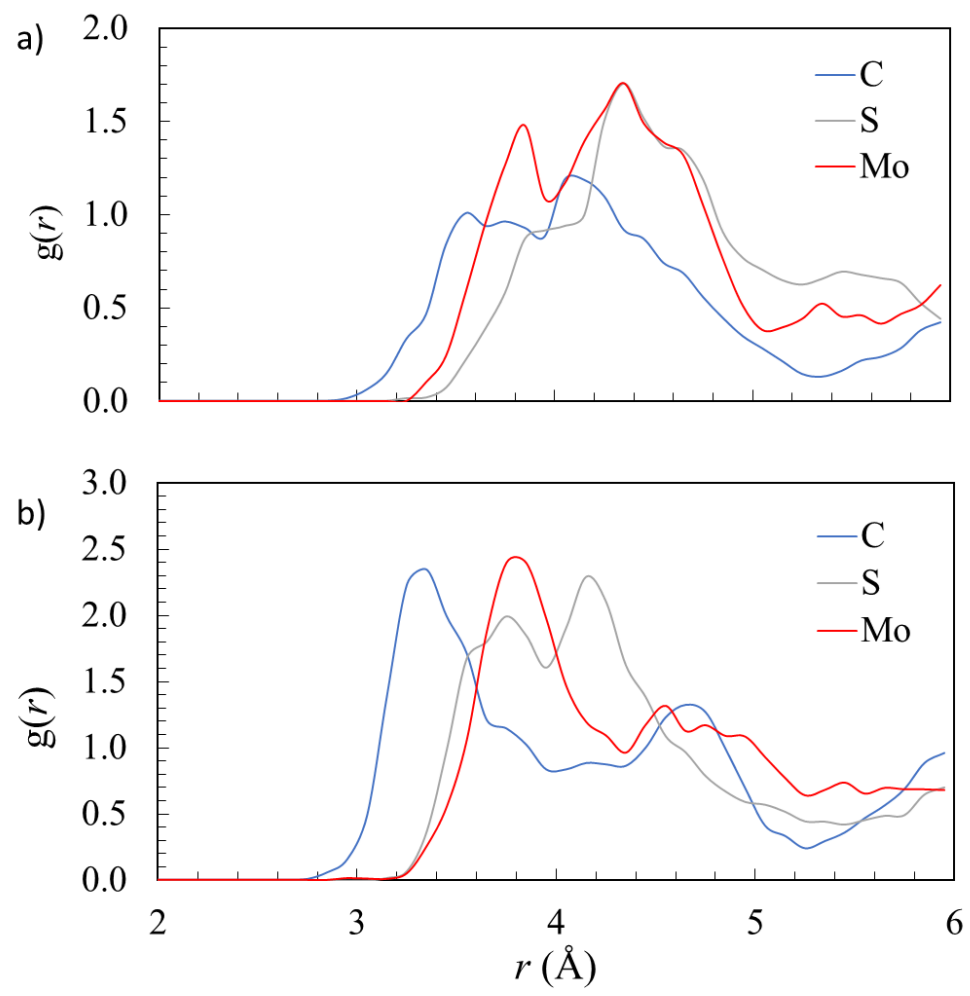

Figure 3. Radial distribution function $X-\mathrm{O}_{\mathrm{w}}$, i.e. from the $X=(\mathrm{C}, \mathrm{S}, \mathrm{Mo})$ atom in the $\left(X \mathrm{O}_{n}\right)^{2-}$ oxyanion to the oxygen atoms $\left(\mathrm{O}_{\mathrm{w}}\right)$ in water molecule, for the simulations of oxyanions substitutions at a) the calcite terrace and b) the calcite step. 
Another significant trend in the exchange energies (Table 2) is that molybdate appears more favourable than sulphate when substituting both surface sites, which is a reversal of the bulk incorporation energy trends. This means that despite greater steric strain caused by molybdate over sulphate, other factors are significant in driving the thermodynamic exchange energy. A possible explanation comes from the different levels of hydration of the substituting ions at the surface. As the molybdate ion protrudes more readily from the surface, aside from reducing lattice strain this also allows for the formation of stronger partial hydration spheres. Hydration sphere formation is favourable and drives the thermodynamic process of substitution. Figure $3 \mathrm{a}$ reports RDF's showing the partial hydration spheres formed around sulphate and molybdate ions substituted at the calcite terrace. Molybdate shows clear first peat at around $3.8 \AA$, while sulphate shows only a very small shoulder at this region. The explanation for this is that the molybdate ion protrudes from the surface more significantly than the sulphate meaning that the central atom $(\mathrm{Mo})$ is closer to the water than the central atom (S) in sulphate, which is more deeply embedded into the lattice. Similar trends are reported in Figure 3b - RDF's showing partial hydration environments for sulphate and molybdate substituted at the calcite step. The molybdate shows a stronger, more well-defined first hydration sphere when compared to sulphate, because it is repelled from the carbonate crystal more strongly and is therefore in closer contact with the surrounding water. This analysis provides more evidence to the notion that the molybdate is more strongly ejected from the terrace while remaining chemically bound. Our proposed mechanism of stronger patrial surface rehydration for molybdate over sulphate also gives another thermodynamic contributor to the relative favourability of molybdate substitution over sulphate at this site.

\section{Conclusions}

We have presented an ab initio molecular dynamics investigation of the incorporation of sulphate and molybdate as substitutional impurities in the bulk and surface of calcite. Results from this investigation indicate a strong tendency for ion exchange at the calcite/water interface regions of the speleothem when compared to exchange with the crystalline bulk. When comparing the incorporation of a given impurity species across the bulk, terrace and step substitution sites, the ability of the solid to accommodate the tetrahedral oxyanion is still the key thermodynamic factor. In order to understand the differences between sulphate and molybdate substitution thermodynamics, we need to pay attention to other factors, for example the partial hydration of the impurity at the calcite surface.

Bulk ion exchange of molybdate with liquid water is more thermodynamically unfavourable than sulphate, which is due to the increased lattice strain caused by the much larger molybdate ion. Exchange at both the terraced and stepped interfaces is more favourable for molybdate than for sulphate, which is a reversal in the trend observed for the two ions in the bulk. We rationalise this trend reversal by considering that the molybdate ion is ejected from the solid surface more significantly due to higher lattice strain, but is able to remain chemically bound to the solid due to its longer $X$-O bonds than sulphate. This proposed mechanism is 
supported by the analysis of radial distribution functions indicating stronger partial rehydration of the molybdate impurity at the surfaces, because the ionic centre (Mo) is less deeply embedded in the carbonate solid, meaning closer proximity to surrounding water than the analogous $\mathrm{S}$ atom. Although our analysis here is based on thermodynamics, the preferential substitution of the anion impurities at the surface and steps of calcite must also have important consequences for the growth kinetics. Calcite crystals growth is dominated by steps dynamics, and the presence of impurities, particularly during extreme events such as volcanic eruptions, at the steps is known to stunt calcite growth. ${ }^{24,45}$ The interplay beyond thermodynamic and kinetic factors in this phenomenon is complex and beyond the scope of the present study.

Our calculations demonstrate and quantify the importance of surfaces and surface defects in the incorporation of oxyanion impurities in calcite. Since Mo and S impurities in speleothem calcites constitute a reliable record of past volcanic activity, the understanding gained of the factors controlling the concentrations of these impurities in calcite will be useful for a quantitative interpretation of speleothem records in the future.

\section{Acknowledgements}

S. D. Midgley acknowledges funding for his PhD studentship from the Natural Environment Research Council through the SCENARIO DTP (grant ref NE/L002566/1). This work made use of ARCHER, the UK's national high-performance computing service, via the UK's HPC Materials Chemistry Consortium, which is funded by EPSRC (EP/R029431), and of the Young supercomputer, via the UK's Materials and Molecular Modelling Hub, which is partially funded by EPSRC (EP/T022213/1).

\section{References}

1. Minnis, P.; Harrison, E. F.; Stowe, L. L.; Gibson, G. G.; Denn, F. M.; Doelling, D. R.; Smith, W. L., Radiative Climate Forcing by the Mount Pinatubo Eruption. Science 1993, 259, 1411.

2. Robock, A.; Free, M. P., Ice Cores as an Index of Global Volcanism from 1850 to the Present. Journal of Geophysical Research: Atmospheres 1995, 100, 11549-11567.

3. Severi, M.; Udisti, R.; Becagli, S.; Stenni, B.; Traversi, R., Volcanic Synchronisation of the Epica-Dc and Taldice Ice Cores for the Last 42 Kyr Bp. Climate of the Past. 2012, 8, 509-517.

4. Yalcin, K.; Wake, C. P.; Kreutz, K. J.; Germani, M. S.; Whitlow, S. I., Ice Core Paleovolcanic Records from the St. Elias Mountains, Yukon, Canada. Journal of Geophysical Research 2007, 112, D08102-D08102.

5. Zielinski, G. A.; Mayewski, P. A.; Meeker, L. D.; Whitlow, S.; Twickler, M. S.; Morrison, M.; Meese, D. A.; Gow, A. J.; Alley, R. B., Record of Volcanism since 7000 B.C. From the Gisp2 Greenland Ice Core and Implications for the Volcano-Climate System. Science (New York, N.Y.) 1994, 264, 948-52.

6. McCarroll, D.; Loader, N. J., Stable Isotopes in Tree Rings. Quaternary Science Reviews 2004, 23, 771801.

7. Voelker, A. H. L., Global Distribution of Centennial-Scale Records for Marine Isotope Stage (Mis) 3: A Database. Quaternary Science Reviews 2002, 21, 1185-1212. 
8. Badertscher, S.; Borsato, A.; Frisia, S.; Cheng, H.; Edwards, R. L.; Tüysüz, O.; Fleitmann, D., Speleothems as Sensitive Recorders of Volcanic Eruptions - the Bronze Age Minoan Eruption Recorded in a Stalagmite from Turkey. Earth and Planetary Science Letters 2014, 392, 58-66.

9. Fairchild, I. J.; Treble, P. C., Trace Elements in Speleothems as Recorders of Environmental Change. Quaternary Science Reviews 2009, 28, 449-468.

10. Finch, A. A.; Shaw, P. A.; Weedon, G. P.; Holmgren, K., Trace Element Variation in Speleothem Aragonite: Potential for Palaeoenvironmental Reconstruction. Earth and Planetary Science Letters 2001, 186, 255-267.

11. Ünal-İmer, E.; Shulmeister, J.; Zhao, J.-X.; Tonguç Uysal, I.; Feng, Y.-X.; Duc Nguyen, A.; Yüce, G., An 80 Kyr-Long Continuous Speleothem Record from Dim Cave, Sw Turkey with Paleoclimatic Implications for the Eastern Mediterranean. Scientific Reports 2015, 5, 13560-13560.

12. Dorale, J. A.; Edwards, R. L.; Calvin-Alexander Jr, E.; Shen, C. C.; Richards, D. A.; Cheng, H., Studies of Cave Sediments; Springer US: Boston, MA, 2004, p 177-197.

13. St Pierre, E.; Zhao, J.-X.; Reed, E., Expanding the Utility of Uranium-Series Dating of Speleothems for Archaeological and Palaeontological Applications. Journal of Archaeological Science 2009, 36, 1416-1423.

14. De Choudens-Sanchez, V.; Gonzalez, L. A., Calcite and Aragonite Precipitation under Controlled Instantaneous Supersaturation: Elucidating the Role of $\mathrm{Caco}_{3}$ Saturation State and $\mathrm{Mg} / \mathrm{Ca}$ Ratio on Calcium Carbonate Polymorphism. Journal of Sedimentary Research 2009, 79, 363-376.

15. Given, R. K.; Wilkinson, B. H., Kinetic Control of Morphology, Composition, and Mineralogy of Abiotic Sedimentary Carbonates. SEPM Journal of Sedimentary Research 1985, Vol. 55, 109-119.

16. Railsback, L. B.; Brook, G. A.; Chen, J.; Kalin, R.; Fleisher, C. J., Environmental Controls on the Petrology of a Late Holocene Speleothem from Botswana with Annual Layers of Aragonite and Calcite. SEPM Journal of Sedimentary Research 1994, Vol. 64A, 147-155.

17. Midgley, S. D.; Taylor, J. O.; Fleitmann, D.; Grau-Crespo, R., Molybdenum and Sulphur Incorporation as Oxyanion Substitutional Impurities in Calcium Carbonate Minerals: A Computational Investigation. Chemical Geology 2020.

18. Blanchard, D. L.; Baer, D. R., The Interactions of Co, Mn and Water with Calcite Surfaces. Surface Science 1992, 276, 27-39.

19. Didymus, J. M.; Oliver, P.; Mann, S.; DeVries, A. L.; Hauschka, P. V.; Westbroek, P., Influence of Low-Molecular-Weight and Macromolecular Organic Additives on the Morphology of Calcium Carbonate. Journal of the Chemical Society, Faraday Transactions 1993, 89, 2891-2900.

20. MacInnis, I. N.; Brantley, S. L., The Role of Dislocations and Surface Morphology in Calcite Dissolution. Geochimica et Cosmochimica Acta 1992, 56, 1113-1126.

21. Gratz, A. J.; Hillner, P. E.; Hansma, P. K., Step Dynamics and Spiral Growth on Calcite. Geochimica et Cosmochimica Acta 1993, 57, 491-495.

22. Liang, Y.; Lea, A. S.; Baer, D. R.; Engelhard, M. H., Structure of the Cleaved $\mathrm{Caco}_{3}(1014)$ Surface in an Aqueous Environment. Surface Science 1996, 351, 172-182. 
23. Davis, K. J.; Dove, P. M.; Yoreo, J. J. D., Resolving the Control of Magnesium on Calcite Growth: Thermodynamic and Kinetic Consequences of Impurity Incorporation for Biomineral Formation. MRS Proceedings 2000, 620, M9.5.1.

24. Wasylenki, L. E.; Dove, P. M.; Wilson, D. S.; De Yoreo, J. J., Nanoscale Effects of Strontium on Calcite Growth: An in Situ Afm Study in the Absence of Vital Effects. Geochimica et Cosmochimica Acta 2005, 69, 3017-3027.

25. Meyer, H. J., The Influence of Impurities on the Growth Rate of Calcite. Journal of Crystal Growth 1984, 66, 639-646.

26. Nielsen, L. C.; De Yoreo, J. J.; DePaolo, D. J., General Model for Calcite Growth Kinetics in the Presence of Impurity Ions. Geochimica et Cosmochimica Acta 2013, 115, 100-114.

27. Paquette, J.; Reeder, R. J., Relationship between Surface Structure, Growth Mechanism, and Trace Element Incorporation in Calcite. Geochimica et Cosmochimica Acta 1995, 59, 735-749.

28. Staudt, W. J.; Reeder, R. J.; Schoonen, M. A. A., Surface Structural Controls on Compositional Zoning of $\mathrm{So}_{4}{ }^{2-}$ and $\mathrm{SeO}_{4}{ }^{2-}$ in Synthetic Calcite Single Crystals. Geochimica et Cosmochimica Acta 1994, 58, 20872098.

29. Markgraf, S.; Reeder, R., High-Temperature Structure Refinements of Calcite and Magnesite. Am. Mineral. 1985, 70, 590-600.

30. de Leeuw, N. H.; Parker, S. C.; Harding, J. H., Molecular Dynamics Simulation of Crystal Dissolution from Calcite Steps. Physical Review B 1999, 60, 13792-13799.

31. The CP2K Developers Group https://www.cp2k.org/ (accessed April 3.

32. Gale, J. D.; Rohl, A. L., The General Utility Lattice Program (Gulp). Molecular Simulation 2003, 29, 291-341.

33. Fisler, D. K.; Gale, J. D.; Cygan, R. T., A Shell Model for the Simulation of Rhombohedral Carbonate Minerals and Their Point Defects. American Mineralogist 2000, 85, 217-224.

34. Romao, C. P., Anisotropic Thermal Expansion in Flexible Materials. Physical Review B 2017, 96, 134113.

35. VandeVondele, J.; Krack, M.; Mohamed, F.; Parrinello, M.; Chassaing, T.; Hutter, J., Quickstep: Fast and Accurate Density Functional Calculations Using a Mixed Gaussian and Plane Waves Approach. Comput Phys Commun 2005, 167, 103-128.

36. VandeVondele, J.; Hutter, J., An Efficient Orbital Transformation Method for Electronic Structure Calculations. J Chem Phys 2003, 118, 4365-4369.

37. Zhang, Y. K.; Yang, W. T., Comment on "Generalized Gradient Approximation Made Simple". Phys Rev Lett 1998, 80, 890-890.

38. Ruiz Pestana, L.; Mardirossian, N.; Head-Gordon, M.; Head-Gordon, T., Ab Initio Molecular Dynamics Simulations of Liquid Water Using High Quality Meta-Gga Functionals. Chemical Science 2017, 8, 3554-3565.

39. Grimme, S.; Ehrlich, S.; Goerigk, L., Effect of the Damping Function in Dispersion Corrected Density Functional Theory. J Comput Chem 2011, 32, 1456-1465. 
40. VandeVondele, J.; Hutter, J., Gaussian Basis Sets for Accurate Calculations on Molecular Systems in Gas and Condensed Phases. J Chem Phys 2007, 127.

41. Goedecker, S.; Teter, M.; Hutter, J., Separable Dual-Space Gaussian Pseudopotentials. Physical Review B 1996, 54, 1703-1710.

42. Nosé, S., A Unified Formulation of the Constant Temperature Molecular Dynamics Methods. The Journal of Chemical Physics 1984, 81, 511-519.

43. Smith, D. W., Ionic Hydration Enthalpies. Journal of Chemical Education 1977, 54, 540.

44. Wang, X.; Toroz, D.; Kim, S.; Clegg, S. L.; Park, G.-S.; Di Tommaso, D., Density Functional Theory Based Molecular Dynamics Study of Solution Composition Effects on the Solvation Shell of Metal Ions. Physical Chemistry Chemical Physics 2020, 22, 16301-16313.

45. de Leeuw, N. H., Molecular Dynamics Simulations of the Growth Inhibiting Effect of $\mathrm{Fe}^{2+}, \mathrm{Mg}^{2+}, \mathrm{Cd}^{2+}$, and $\mathrm{Sr}^{2+}$ on Calcite Crystal Growth. The Journal of Physical Chemistry B 2002, 106, 5241-5249. 